\title{
Synthesis of the C1-C27 Fragment of Stambomycin D Validates Modular Polyketide Synthase-Based Stereochemical Assignments
}

\author{
Jieyan Lim,,${ }^{\ddagger}$ Venkaiah Chintalapudi, ${ }^{+}$Haraldur G. Gudmundsson, Minh Tran, Alice Bernasconi, \\ Araceli Blanco, Lijiang Song, Gregory L. Challis, and Edward A. Anderson*
}

Cite This: Org. Lett. 2021, 23, 7439-7444

Read Online

\section{ACCESS |}

Џ Metrics \& More

| 回 Article Recommendations

Supporting Information

ABSTRACT: The stambomycins are a family of bioactive macrolides isolated from Streptomyces ambofaciens. Aside from two stereocenters installed through cytochrome P450 oxidations, their stereochemistry has been predicted by sequence analysis of the polyketide synthase. We report a synthesis of the $\mathrm{C} 1-\mathrm{C} 27$ fragment of stambomycin $\mathrm{D}$, the spectroscopic data of which correlates well with that of the natural product, further validating predictive sequence analysis as a powerful tool for stereochemical assignment of complex polyketide natural products.

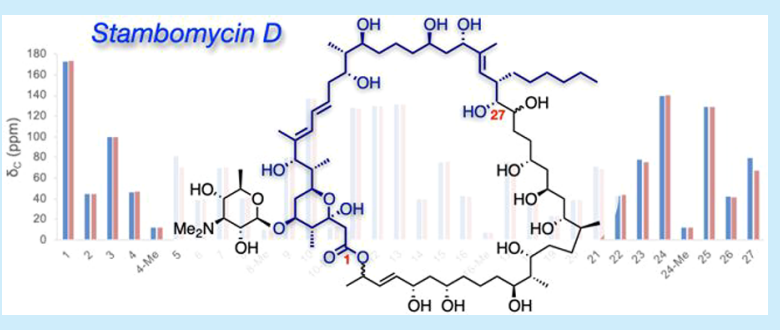

tereochemical determination is a key element in natural $\checkmark$ product discovery, as bioactivity is often intrinsically linked to stereochemistry. It can also be one of the most challenging aspects, especially for polyketides where conformational flexibility and noncrystallinity render conclusive assignment challenging using NMR-based methods or X-ray crystallography. ${ }^{1-3}$ Computational approaches using NMR parameters are emerging as reliable tools ${ }^{4,5}$ but are unsuitable when individual stereoclusters are "insulated" by regions of flexible nonfunctionalized carbon chains, or by rigid (poly)alkene regions. NMR spectroscopy can equally be ambiguous for certain stereoclusters/conformations or complicated by overlapping signals in more complex natural products, rendering the extraction of coupling constants or nOes highly challenging and ultimately not definitive. This uncertainty provides a significant obstacle for synthesis and applications. ${ }^{6}$

Advances in bioinformatics have enabled the application of predictive sequence analysis of biosynthetic enzymes not only in the discovery of natural products but also in their structural and stereochemical determination. ${ }^{7-11}$ One example is the stambomycins, a family of 51-membered glycosylated macrolides discovered by Challis, Aigle, and co-workers in 2011 (Figure 1a). ${ }^{12}$ These were identified as the metabolic products of a modular polyketide synthase (PKS) in Streptomyces ambofaciens through a genomics-driven approach involving rational genetic manipulation to induce transcription of the biosynthetic genes, which are poorly expressed in laboratory cultures. Four members of the family (A-D) were identified, differing at the $\mathrm{C} 26$ side chain, all of which showed potent antibacterial and antitumor activity. The planar structures and stereochemistry of the stambomycins were predicted via sequence analysis of the modular PKS responsible for their biosynthesis, ${ }^{12-14}$ with the exception of the C28 and C50 stereocenters, which are of non-PKS origin. ${ }^{15}$ Notably, the

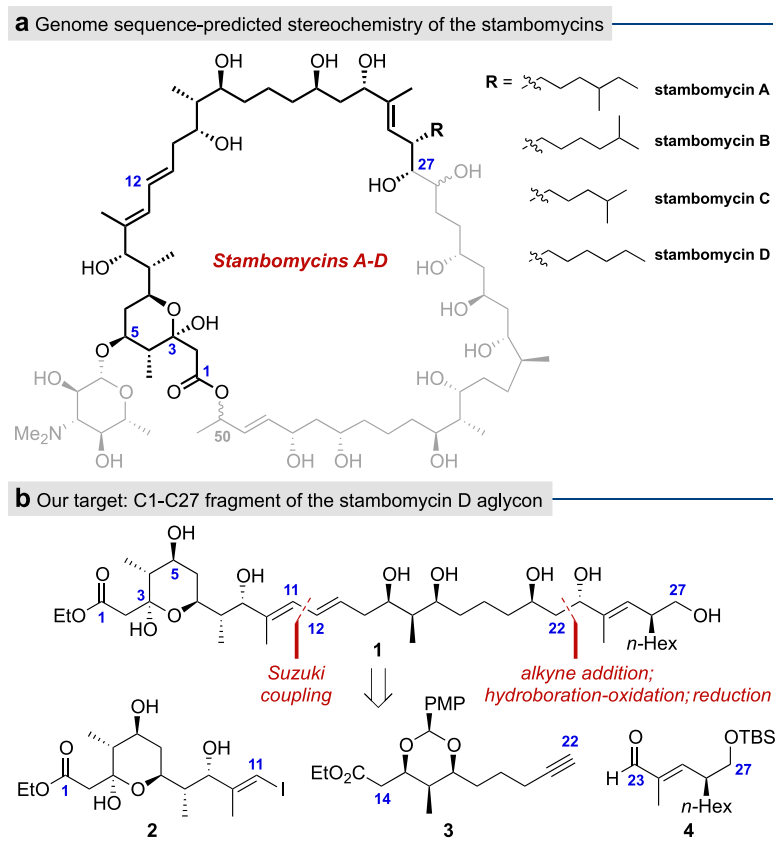

Figure 1. (a) Stambomycins $A-D$ and (b) the $\mathrm{C} 1-\mathrm{C} 27$ fragment and planned retrosynthesis.

Received: August 9, 2021

Published: September 8, 2021 
stambomycins are one of the earliest structurally complex polyketides for which predictive sequence analysis was employed for stereochemical assignment, and remain one of the most elaborate examples to date. ${ }^{16}$

While the predicted planar structures of the stambomycins have been confirmed by NMR spectroscopy, their stereochemistry remains to be unequivocally confirmed. This inspired our interest in stambomycin D: a synthesis of this molecule would represent a powerful validation of sequencebased polyketide stereochemical assignment, and one that could offer a rapid and complementary approach to traditional NMR-based methods. Here, we report the synthesis of the $\mathrm{C} 1-\mathrm{C} 27$ aglycon fragment of stambomycin $\mathrm{D}$ and its comparison with the natural macrocycle. The excellent agreement between the synthetic and natural material supports the sequence-based stereochemical assignment in this region.

We envisioned that the northern and southern hemispheres of the stambomycin D aglycon would make ideal targets to establish a synthetic strategy for the entire molecule and allow a preliminary comparison of NMR data to support the predicted stereochemistry. To avoid the uncertainty of the C28 stereocenter, our initial target for the northern hemisphere consisted of the $\mathrm{C} 1-\mathrm{C} 27$ fragment 1 (Figure $1 \mathrm{~b}$ ). Retrosynthetically, 1 could be disconnected at the $\mathrm{C} 11-\mathrm{C} 12$ bond to reveal $\mathrm{C} 1-\mathrm{C} 11$ alkenyl iodide 2 , which could be coupled to a vinyl organometallic at C12, for example by Suzuki coupling. Disconnection at the $\mathrm{C} 22-\mathrm{C} 23$ bond reveals $\mathrm{C} 13-\mathrm{C} 22$ fragment 3 (in which the required boronic ester could be derived from manipulation of the ester group) and C23-C27 fragment 4. Union of the latter two fragments could be achieved by asymmetric alkyne addition of 3 to 4 , followed by Hoveyda hydroboration/oxidation ${ }^{17}$ and reduction of the resulting propargylic alcohol to install the desired 1,3-antidiol at C21/C23.

Synthesis of the C1-C11 fragment 2 (Scheme 1) commenced with an enantio- and diastereoselective Leighton crotylation $^{18}$ of aldehyde 5 with cis-crotyltrichlorosilane 7 to give homoallylic alcohol $\mathbf{8}$ in $87 \%$ yield ( $89 \% e e, 15: 1 d r)$. Cross metathesis of $\mathbf{8}$ with methyl acrylate afforded $\alpha, \beta$ unsaturated ester 9 (90\%), which was subjected to EvansPrunet acetalization ${ }^{19}$ to obtain acetal $\mathbf{1 0}$ in $33 \%$ yield. Formation of this acetal appeared to be in an unfavorable equilibrium with the retro-Michael reaction, as the cyclization failed to reach completion even with extended reaction times; interestingly, the recovered starting material bore mainly a $Z$ alkene. This problem is attributed to the presence of the C8 (R)-methyl group, which must adopt an axial position in the six-membered cyclic acetal. Following a DIBALH reduction of the ester in 10, a second Leighton crotylation was carried out on the resulting aldehyde, giving homoallylic alcohol 11 in $70 \%$ yield $(10: 1 d r)$. Protection of the alcohol as the PMB ether and subsequent oxidative cleavage of the terminal alkene afforded aldehyde 12. A Mukaiyama aldol reaction of 12 with silyl ketene acetal 13 then gave the corresponding $\beta$-hydroxy ester $(85 \%)$, which after oxidation of the alcohol, furnished $\beta$-keto ester 14 in $87 \%$ yield.

We expected that deprotection of the acetal under acidic conditions would also promote spontaneous cyclization of the resulting $\mathrm{C} 7$ hydroxyl group onto the $\mathrm{C} 3$ ketone to form the desired tetrahydropyran. This step proved unexpectedly challenging as the acetal was surprisingly robust; conditions that allowed for full conversion of the starting material also resulted in significant degradation and the formation of an
Scheme 1. Synthesis of C1-C11 Fragment 2

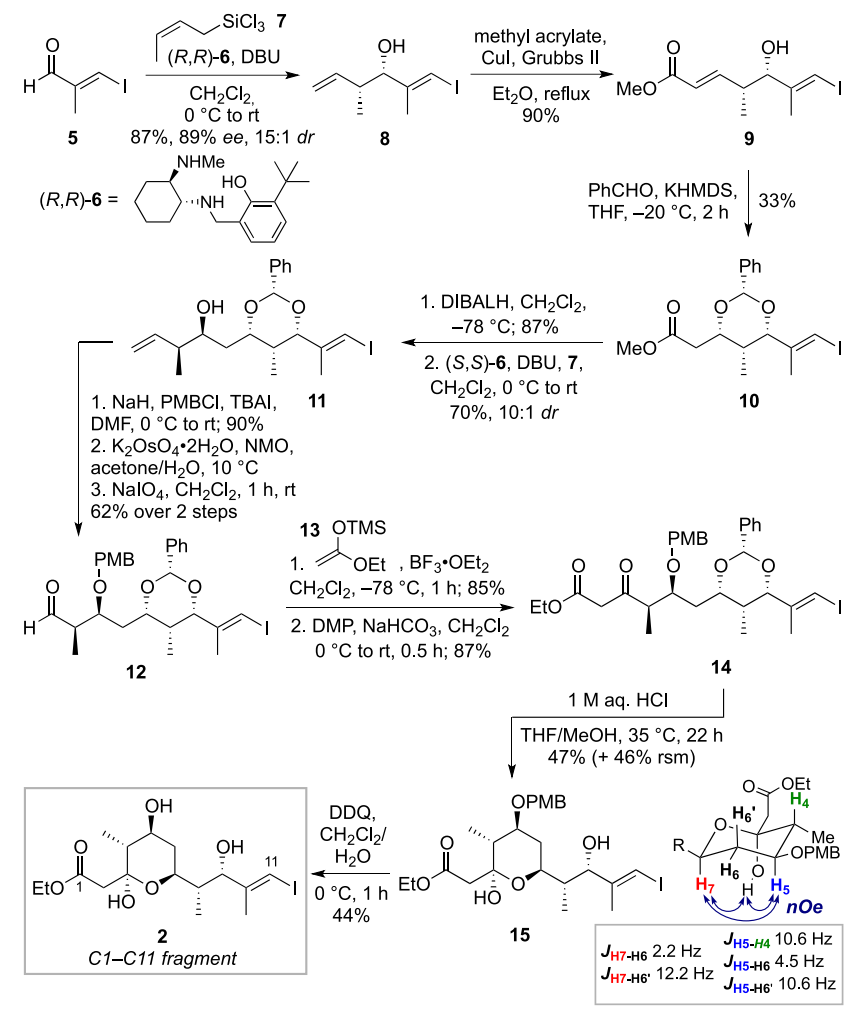

unidentified side product which was difficult to separate from the product 15. Various deprotection conditions were tested to achieve an optimal balance between conversion of the starting material and product formation, most of which involved different concentrations of aqueous $\mathrm{HCl}$ in $\mathrm{MeOH} / \mathrm{THF}$, as this acid was observed to give a relatively clean reaction. After fine-tuning the solvent ratio, temperature, and reaction time, it was found that use of $1.0 \mathrm{M}$ aqueous $\mathrm{HCl}$ in $\mathrm{MeOH} / \mathrm{THF}$ (1:1) at $35^{\circ} \mathrm{C}$ for $22 \mathrm{~h}$ gave the desired tetrahydropyran 15 in $47 \%$ yield, with $46 \%$ recovered starting material. NOESY correlations and coupling constant analysis confirmed the relative stereochemistry of the various substituents on the 6membered ring. Finally, PMB deprotection afforded the $\mathrm{C} 1-$ C11 fragment 2 in $44 \%$ yield (12 steps from 5 ).

Synthesis of the C13-C22 fragment 3 (Scheme 2a) began with 5-hexynal 16. A Leighton crotylation ${ }^{18}$ was again employed to set the two adjacent stereocenters in homoallylic alcohol $17(82 \%, 92 \% e e$, > 20:1 dr). Adopting a similar strategy to that used for fragment 2 , alcohol 17 was protected as the PMB ether, with subsequent oxidative cleavage of the terminal alkene affording aldehyde 18. A Mukaiyama aldol reaction of 18 with silyl ketene acetal 13 gave $\beta$-hydroxy ester 19 in $86 \%$ yield $(5: 1 d r)$; the stereochemistry of the alcohol was confirmed by Mosher ester analysis. ${ }^{20}$ Finally, treatment of 19 with DDQ under anhydrous conditions resulted in the formation of the 1,3-PMP acetal, giving C13-C22 fragment 3 in $55 \%$ yield after removal of the minor diastereomer ( 6 steps from 16).

Attention now turned to the construction of the C23-C27 aldehyde 4 (Scheme 2b). To install the hexyl-bearing stereocenter in this fragment, an enantioselective organocatalytic aldol reaction ${ }^{21}$ of octanal and formaldehyde was employed at the outset. This gave a lactol intermediate, which was subjected to a Wittig olefination to obtain enoate 21 in 
Scheme 2. (a) Synthesis of C13-C22 Fragment 3 and (b) Synthesis of C23-C27 Fragment 4

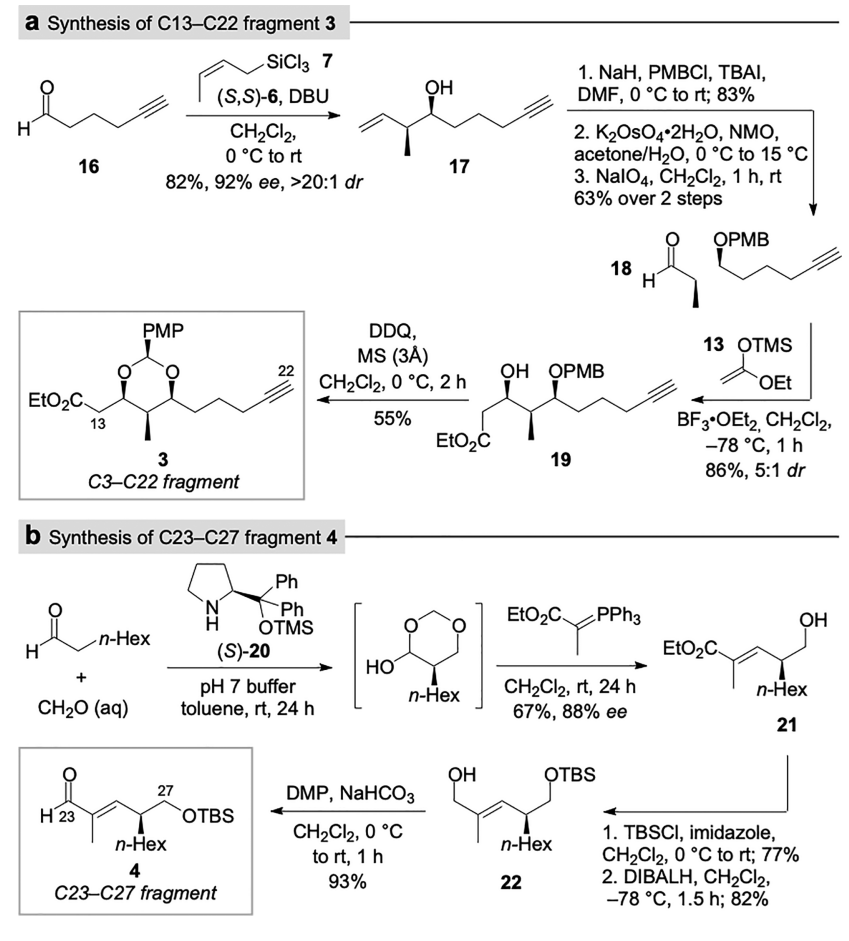

$67 \%$ yield $(88 \% e e)$. Protection of the alcohol as the TBS ether (77\%) and DIBALH reduction of the ester (82\%) gave alcohol 22. Oxidation of alcohol 22 then afforded the C23-C27 aldehyde 4 in $93 \%$ yield.

With fragments 2-4 in hand, we proceeded to combine them toward the full $\mathrm{C} 1-\mathrm{C} 27$ fragment 1 (Scheme 3). First, a diastereoselective alkynylzinc addition ${ }^{22}$ of 3 to 4 afforded propargylic alcohol 23 in $62 \%$ yield $(11: 1 d r)$, with Mosher ester analysis confirming the stereochemistry of the alcohol. Hydroboration/oxidation of 23 employing a modification ${ }^{23}$ of Hoveyda's conditions ${ }^{17}$ gave $\beta$-hydroxy ketone $\mathbf{2 4}$ in $80 \%$ yield. Following an Evans-Saksena reduction ${ }^{24}$ of the $\beta$ hydroxy ketone (>20:1 $d r$ ), the resulting 1,3-anti-diol was protected as the acetonide (25), which moreover served to confirm its stereochemistry through the Rychnovsky method. ${ }^{25}$ A DIBALH reduction of the ester in $\mathbf{2 5}$ afforded the aldehyde, which was then alkynylated using the Ohira-Bestmann reagent. During alkynylation, it was observed that the PMP acetal was prone to ring-opening, presumably via enolization of the adjacent aldehyde under the mildly basic reaction conditions $\left(\mathrm{K}_{2} \mathrm{CO}_{3}\right)$. This resulted in the formation of a side product which not only lowered the yield of the alkyne (26) but also led to problems with purification. It was eventually found that use of an excess of the Ohira-Bestmann reagent overcame this problem, enabling alkyne 26 to be obtained in $81 \%$ yield.

Following Zr-mediated hydroboration ${ }^{26}$ of alkyne 26, the resulting vinylboronic ester 27 was coupled with $\mathrm{C} 1-\mathrm{C} 11$ fragment 2 via a Suzuki coupling. A variety of reaction conditions were screened, but use of $\mathrm{Tl}_{2} \mathrm{CO}_{3}{ }^{27}$ was found to be essential for reaction success, giving the complete $\mathrm{C} 1-\mathrm{C} 27$ framework $\mathbf{2 8}$ in $53 \%$ yield. Deprotection of $\mathbf{2 8}$ proved nontrivial, as the $\mathrm{C} 10-\mathrm{C} 13$ 1,3-diene was observed to be highly acid-sensitive and prone to degradation, potentially via acid-promoted cyclization of the $\mathrm{C} 17$ hydroxyl group. After much experimentation, we found that deprotection could be achieved using $0.1 \mathrm{M}$ aqueous $\mathrm{HCl}$ in $\mathrm{MeOH} / \mathrm{THF}$ without degradation of the diene. Lower acid concentrations of 0.05 and $0.02 \mathrm{M}$ could also be used, although longer reaction times were required. Treatment of 28 with $0.1 \mathrm{M}$ aqueous $\mathrm{HCl}$ in $\mathrm{MeOH} / \mathrm{THF}$ for three hours at room temperature thus afforded $\mathrm{C} 1-\mathrm{C} 27$ fragment 1 in $41 \%$ yield.

Having obtained $\mathrm{C} 1-\mathrm{C} 27$ fragment 1 , we were inspired to compare its NMR spectra with the corresponding NMR data of stambomycin D. To our delight, the data for 1 showed an excellent match with the reported ${ }^{12}$ data for stambomycin D (Figure 2 and Supporting Information). Although slight discrepancies existed, this is not unexpected due to potential conformational differences between the acyclic fragment and

Scheme 3. Completion of C1-C27 Fragment 1

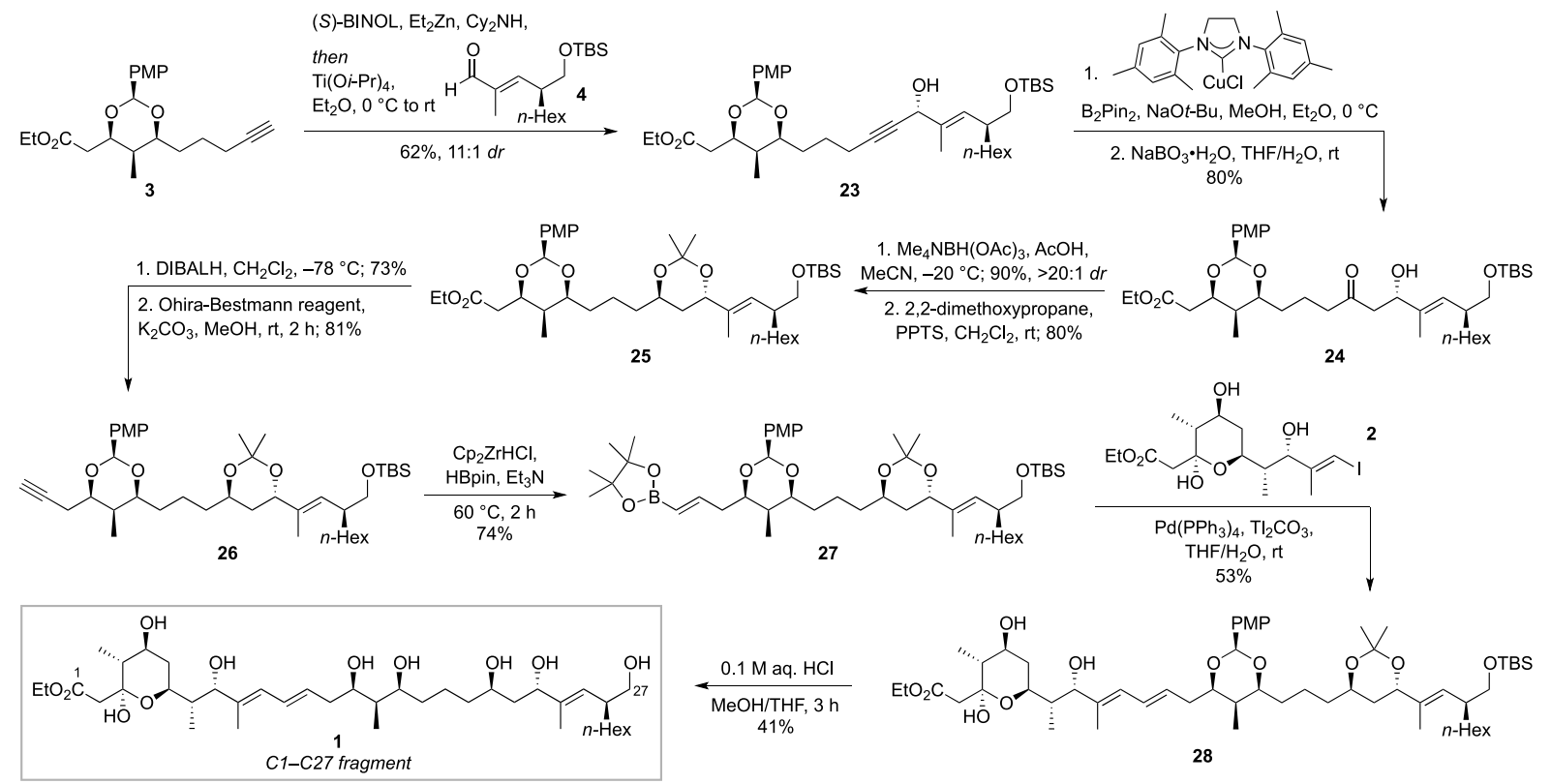




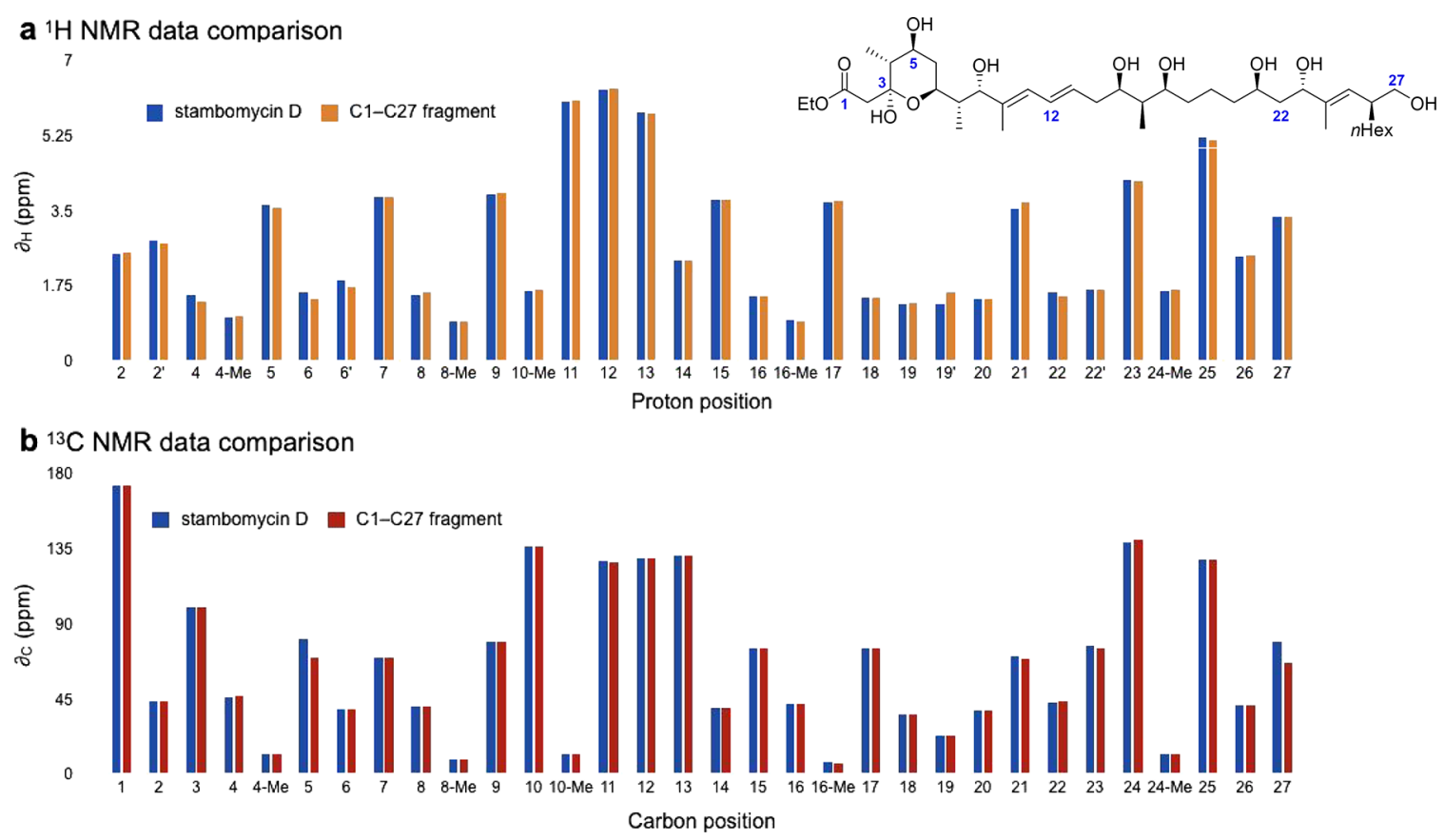

Figure 2. Comparison of (a) ${ }^{1} \mathrm{H}$ NMR and (b) ${ }^{13} \mathrm{C}$ NMR data of stambomycin D and $\mathrm{C} 1-\mathrm{C} 27$ fragment 1.

the cyclic macrolide. For example, the acyclic fragment contains a free hydroxyl at C5, whereas in the macrolide this oxygen atom is attached to the amino sugar mycaminose; in addition, the acyclic fragment is truncated at $\mathrm{C} 27$, as compared to the macrolide. These differences were therefore reflected in discrepancies in the ${ }^{13} \mathrm{C}$ NMR data of $\mathrm{C5}$ and C27. An additional discrepancy was noted at the $\mathrm{C} 19$ protons; reexamination of the spectroscopic data for the natural product confirmed these signals should be reassigned. Overall, there is good agreement in the ${ }^{1} \mathrm{H}$ and ${ }^{13} \mathrm{C}$ NMR data between the $\mathrm{C} 1-\mathrm{C} 27$ fragment and stambomycin $\mathrm{D}$, supporting the stereochemical assignment of this region of the natural product.

In summary, we have synthesized the $\mathrm{C} 1-\mathrm{C} 27$ "northern" fragment of the stambomycin D aglycon. Comparison of NMR data of this fragment with the reported data of stambomycin D showed good agreement between the two, providing preliminary proof of the accuracy of the sequence-based stereochemical assignment of the macrolide.

\section{ASSOCIATED CONTENT}

\section{SI Supporting Information}

The Supporting Information is available free of charge at https://pubs.acs.org/doi/10.1021/acs.orglett.1c02650.

Experimental procedures and characterization data for novel compounds (PDF)

\section{AUTHOR INFORMATION}

\section{Corresponding Author}

Edward A. Anderson - Chemistry Research Laboratory, University of Oxford, Oxford OX1 3TA, U.K.; (1) orcid.org/ 0000-0002-4149-0494; Email: edward.anderson@ chem.ox.ac.uk

\section{Authors}

Jieyan Lim - Chemistry Research Laboratory, University of Oxford, Oxford OX1 3TA, U.K.

Venkaiah Chintalapudi - Chemistry Research Laboratory, University of Oxford, Oxford OX1 3TA, U.K.

Haraldur G. Gudmundsson - Chemistry Research Laboratory, University of Oxford, Oxford OX1 3TA, U.K.

Minh Tran - Chemistry Research Laboratory, University of Oxford, Oxford OX1 3TA, U.K.

Alice Bernasconi - Sezione Chimica Generale e Organica "A. Marchesini”, Università degli Studi di Milano, 20133 Milano, Italy

Araceli Blanco - Departamento de Química Orgánica, Facultad de Ciencias Químicas, Universidad de Salamanca, 37008 Salamanca, Spain

Lijiang Song - Department of Chemistry and Warwick Integrative Synthetic Biology Centre, University of Warwick, Coventry CV4 7AL, U.K.

Gregory L. Challis - Department of Chemistry and Warwick Integrative Synthetic Biology Centre, University of Warwick, Coventry CV4 7AL, U.K.; Department of Biochemistry and Molecular Biology and ARC Centre of Excellence for Innovations in Peptide and Protein Science, Biomedicine Discovery Institute, Monash University, Clayton, Victoria 3800, Australia; orcid.org/0000-0001-5976-3545

Complete contact information is available at: https://pubs.acs.org/10.1021/acs.orglett.1c02650

\section{Author Contributions}

${ }^{\ddagger}$ J.L. and V.C. contributed equally to this work. Notes

The authors declare the following competing financial interest(s): G.L.C. is a nonexecutive codirector of a Erebagen, Ltd. The other authors declare no competing interests. 


\section{ACKNOWLEDGMENTS}

We thank the Agency for Science, Technology and Research (A*STAR) for a National Science Scholarship (J.L.), the Marie Skłodowska-Curie actions for an Individual Fellowship (V.C., GA No 702385), and the Leverhulme Trust for funding (H.G.G., RPG-2015-003). We are grateful to Jonathan Yong (University of Oxford) for his help in acquiring NMR spectra of 1. E.A.A. thanks the EPSRC for additional support (EP/ S013172/1).

\section{REFERENCES}

(1) Matsumori, N.; Kaneno, D.; Murata, M.; Nakamura, H.; Tachibana, K. Stereochemical Determination of Acyclic Structures Based on Carbon-Proton Spin-Coupling Constants. A Method of Configuration Analysis for Natural Products. J. Org. Chem. 1999, 64, 866-876.

(2) (a) Kobayashi, Y.; Lee, J.; Tezuka, K.; Kishi, Y. Toward Creation of a Universal NMR Database for the Stereochemical Assignment of Acyclic Compounds: The Case of Two Contiguous Propionate Units. Org. Lett. 1999, 1, 2177-2180. (b) Lee, J.; Kobayashi, Y.; Tezuka, K.; Kishi, Y. Toward Creation of a Universal NMR Database for the Stereochemical Assignment of Acyclic Compounds: Proof of Concept. Org. Lett. 1999, 1, 2181-2184. (c) Kobayashi, Y.; Hayashi, N.; Tan, C.-H.; Kishi, Y. Toward the Creation of NMR Databases in Chiral Solvents for Assignments of Relative and Absolute Stereochemistry: Proof of Concept. Org. Lett. 2001, 3, 2245-2248. (d) Fleury, E.; Lannou, M.-I.; Bistri, O.; Sautel, F.; Massiot, G.; Pancrazi, A.; Ardisson, J. Advances in the Universal NMR Database: Toward the Determination of the Relative Configurations of Large Polyproprionates. Eur. J. Org. Chem. 2009, 2009, 4992-5001.

(3) For reviews on NMR and related methods for stereochemical determination, see: (a) Menche, D. New methods for stereochemical determination of complex polyketides: configurational assignment of novel metabolites from myxobacteria. Nat. Prod. Rep. 2008, 25, 905918. (b) Menna, M.; Imperatore, C.; Mangoni, A.; Della Sala, G.; Taglialatela-Scafati, O. Challenges in the configuration assignment of natural products. A case-selective perspective. Nat. Prod. Rep. 2019, 36, 476-489.

(4) (a) Smith, S. G.; Goodman, J. M. Assigning the Stereochemistry of Pairs of Diastereoisomers Using GIAO NMR Shift Calculation. J. Org. Chem. 2009, 74, 4597-4607. (b) Smith, S. G.; Goodman, J. M. Assigning Stereochemistry to Single Diastereoisomers by GIAO NMR Calculation: The DP4 Probability. J. Am. Chem. Soc. 2010, 132, 12946-1259. (c) Zanardi, M. M.; Sarotti, A. M. GIAO C-H COSY Simulations Merged with Artificial Neural Networks Pattern Recognition Analysis. Pushing the Structural Validation a Step Forward. J. Org. Chem. 2015, 80, 9371-9378. (d) Ermanis, K.; Parkes, K. E. B.; Agback, T.; Goodman, J. M. The optimal DFT approach in DP4 NMR structure analysis - pushing the limits of relative configuration elucidation. Org. Biomol. Chem. 2019, 17, $5886-5890$

(5) (a) Marcarino, M. O.; Zanardi, M. M.; Cicetti, S.; Sarotti, A. M. NMR Calculations with Quantum Methods: Development of New Tools for Structural Elucidation and Beyond. Acc. Chem. Res. 2020, 53, 1922-1932. (b) Marcarino, M. O.; Cicetti, S.; Zanardi, M. M.; Sarotti, A. M. A critical review on the use of DP4+ in the structural elucidation of natural products: the good, the bad and the ugly. A practical guide. Nat. Prod. Rep. 2021, DOI: 10.1039/D1NP00030F.

(6) For examples, see: (a) Heinrich, M.; Murphy, J. J.; Ilg, M. K.; Letort, A.; Flasz, J.; Philipps, P.; Fürstner, A. Total Synthesis of Putative Chagosensine. Angew. Chem., Int. Ed. 2018, 57, 1357513581. (b) Han, B. Y.; Lam, N. Y. S.; MacGregor, C. I.; Goodman, J. M.; Paterson, I. A synthesis-enabled relative stereochemical assignment of the $\mathrm{C} 1-\mathrm{C} 28$ region of hemicalide. Chem. Commun. 2018, 54, 3247-3250. (c) Paul, D.; Kundu, A.; Saha, S.; Goswami, R. K. Total synthesis: the structural confirmation of natural products. Chem. Commun. 2021, 57, 3307-3322. (d) Fuwa, H. Structure determi- nation, correction, and disproof of marine macrolide natural products by chemical synthesis. Org. Chem. Front. 2021, 8, 3990-4023.

(7) (a) Challis, G. L. Genome Mining for Novel Natural Product Discovery. J. Med. Chem. 2008, 51, 2618-2628. (b) Rutledge, P. J.; Challis, G. L. Discovery of microbial natural products by activation of silent biosynthetic gene clusters. Nat. Rev. Microbiol. 2015, 13, 509523.

(8) Bachmann, B. O.; Van Lanen, S. G.; Baltz, R. H. Microbial genome mining for accelerated natural products discovery: is a renaissance in the making? J. Ind. Microbiol. Biotechnol. 2014, 41, $175-184$.

(9) Ziemert, N.; Alanjary, M.; Weber, T. The evolution of genome mining in microbes - a review. Nat. Prod. Rep. 2016, 33, 988-1005.

(10) Boddy, C. N. Bioinformatics tools for genome mining of polyketide and non-ribosomal peptides. J. Ind. Microbiol. Biotechnol. 2014, 41, 443-450.

(11) Helfrich, E. J. N.; Reiter, S.; Piel, J. Recent advances in genomebased polyketide discovery. Curr. Opin. Biotechnol. 2014, 29, 107115 .

(12) Laureti, L.; Song, L.; Huang, S.; Corre, C.; Leblond, P.; Challis, G. L.; Aigle, B. Identification of a bioactive 51-membered macrolide complex by activation of a silent polyketide synthase in Streptomyces ambofaciens. Proc. Natl. Acad. Sci. U. S. A. 2011, 108, 6258-6263.

(13) Aigle, B.; Challis, G.; Laureti, L.; Song, L.; Leblond, P. Stambomycin and Derivatives, Their Production and Their Use as Drugs. US20120142622A1, June 7, 2012.

(14) (a) Keatinge-Clay, A. T. Stereocontrol within polyketide assembly lines. Nat. Prod. Rep. 2016, 33, 141-149. (b) Keatinge-Clay, A. T. A Tylosin Ketoreductase Reveals How Chirality Is Determined in Polyketides. Chem. Biol. 2007, 14, 898-908. (c) Kwan, D. H.; Sun, Y.; Schulz, F.; Hong, H.; Popovic, B.; Sim-Stark, J. C. C.; Haydock, S. F.; Leadlay, P. F. Prediction and Manipulation of the Stereochemistry of Enoylreduction in Modular Polyketide Synthases. Chem. Biol. 2008, 15, 1231-1240.

(15) Song, L.; Laureti, L.; Corre, C.; Leblond, P.; Aigle, B.; Challis, G. L. Cytochrome P450-mediated hydroxylation is required for polyketide macrolactonization in stambomycin biosynthesis. J. Antibiot. 2014, 67, 71-76.

(16) For selected recent examples where bioinformatics was used in the stereochemical determination of polyketides, see: (a) Hu, Y.; Wang, M.; Wu, C.; Tan, Y.; Li, J.; Hao, X.; Duan, Y.; Guan, Y.; Shang, X.; Wang, Y.; Xiao, C.; Gan, M. Identification and Proposed Relative and Absolute Configurations of Niphimycins C-E from the MarineDerived Streptomyces sp. IMB7-145 by Genomic Analysis. J. Nat. Prod. 2018, 81, 178-187. (b) Kim, M. C.; Machado, H.; Jang, K. H.; Trzoss, L.; Jensen, P. R.; Fenical, W. Integration of Genomic Data with NMR Analysis Enables Assignment of the Full Stereostructure of Neaumycin B, a Potent Inhibitor of Glioblastoma from a MarineDerived Micromonospora. J. Am. Chem. Soc. 2018, 140, 10775-10784. (c) Pérez-Victoria, I.; Oves-Costales, D.; Lacret, R.; Martín, J.; Sánchez-Hidalgo, M.; Díaz, C.; Cautain, B.; Vicente, F.; Genilloud, O.; Reyes, F. Structure elucidation and biosynthetic gene cluster analysis of caniferolides A-D, new bioactive 36-membered macrolides from the marine-derived Streptomyces caniferus CA-271066. Org. Biomol. Chem. 2019, 17, 2954-2971. (d) Kim, M. C.; Winter, J. M.; Cullum, R.; Li, Z.; Fenical, W. Complementary Genomic, Bioinformatics, and Chemical Approaches Facilitate the Absolute Structure Assignment of Ionostatin, a Linear Polyketide from a Rare Marine-Derived Actinomycete. ACS Chem. Biol. 2020, 15, 25072515. (e) Low, Z. J.; Xiong, J.; Xie, Y.; Ma, G.-L.; Saw, H.; Thi Tran, H.; Wong, S. L.; Pang, L. M.; Fong, J.; Lu, P.; Hu, J.-F.; Liang, Y.; Miao, Y.; Liang, Z.-X. Discovery, biosynthesis and antifungal mechanism of the polyene-polyol meijiemycin. Chem. Commun. 2020, 56, 822-825.

(17) Jang, H.; Zhugralin, A. R.; Lee, Y.; Hoveyda, A. H. Highly Selective Methods for Synthesis of Internal $(\alpha$ - $)$ Vinylboronates through Efficient NHC-Cu-Catalyzed Hydroboration of Terminal Alkynes. Utility in Chemical Synthesis and Mechanistic Basis for Selectivity. J. Am. Chem. Soc. 2011, 133, 7859-7871. 
(18) Suen, L. M.; Steigerwald, M. L.; Leighton, J. L. A new and more powerfully activating diamine for practical and scalable enantioselective aldehyde crotylsilylation reactions. Chem. Sci. 2013, 4, 24132417.

(19) Evans, D. A.; Gauchet-Prunet, J. A. Diastereoselective Synthesis of Protected Syn 1,3-Diols by Base-Catalyzed Intramolecular Conjugate Addition of Hemiacetal-Derived Alkoxide Nucleophiles. J. Org. Chem. 1993, 58, 2446-2453.

(20) (a) Dale, J. A.; Mosher, H. S. Nuclear Magnetic Resonance Enantiomer Reagents. Configurational Correlations via Nuclear Magnetic Resonance Chemical Shifts of Diastereomeric Mandelate, $O$-Methylmandelate, and $\alpha$-Methoxy- $\alpha$-trifluoromethylphenylacetate (MTPA) Esters. J. Am. Chem. Soc. 1973, 95, 512-519. (b) Ohtani, I.; Kusumi, T.; Kashman, Y.; Kakisawa, H. High-Field FT NMR Application of Mosher's Method. The Absolute Configurations of Marine Terpenoids. J. Am. Chem. Soc. 1991, 113, 4092-4096.

(21) Boeckman, R. K., Jr.; Biegasiewicz, K. F.; Tusch, D. J.; Miller, J. R. Organocatalytic Enantioselective $\alpha$-Hydroxymethylation of Aldehydes: Mechanistic Aspects and Optimization. J. Org. Chem. 2015, 80, 4030-4045.

(22) Chen, W.; Tay, J.-H.; Ying, J.; Yu, X.-Q.; Pu, L. Catalytic Asymmetric Enyne Addition to Aldehydes and $\mathrm{Rh}(\mathrm{I})$-Catalyzed Stereoselective Domino Pauson-Khand/[4 +2$]$ Cycloaddition. J. Org. Chem. 2013, 78, 2256-2265.

(23) Park, J. K.; Ondrusek, B. A.; McQuade, D. T. Regioselective Catalytic Hydroboration of Propargylic Species Using $\mathrm{Cu}(\mathrm{I})-\mathrm{NHC}$ Complexes. Org. Lett. 2012, 14, 4790-4793.

(24) Evans, D. A.; Chapman, K. T.; Carreira, E. M. Directed Reduction of $\beta$-Hydroxy Ketones Employing Tetramethylammonium Triacetoxyborohydride. J. Am. Chem. Soc. 1988, 110, 3560-1578.

(25) Rychnovsky, S. D.; Skalitzky, D. J. Stereochemistry of Alternating Polyol Chains: ${ }^{13} \mathrm{C}$ NMR Analysis of 1,3-Diol Acetonides. Tetrahedron Lett. 1990, 31, 945-948.

(26) Wang, Y. D.; Kimball, G.; Prashad, A. S.; Wang, Y. Zr-Mediated hydroboration: stereoselective synthesis of vinyl boronic esters. Tetrahedron Lett. 2005, 46, 8777-8780.

(27) Uenishi, J.; Beau, J.-M.; Armstrong, R. W.; Kishi, Y. Dramatic Rate Enhancement of Suzuki Diene Synthesis: Its Application to Palytoxin Synthesis. J. Am. Chem. Soc. 1987, 109, 4756-4758. 